\title{
Notas sobre el uso de ecosistemas subterráneos por murciélagos en el Parque Nacional Tingo María, Huánuco, Perú
}

\author{
Diego Zavala*iD \\ 1 Museo de Historia Natural de la Universidad Nacional de San Agustín (MUSA). Av. Daniel Alcides Carrión S/N, Arequipa, \\ Perú. \\ *Correspondencia: zav3.diego@gmail.com
}

\section{Resumen}

En el Perú existe poca información sobre la biodiversidad de los ecosistemas subterráneos, particularmente de las cuevas. En este trabajo registré qué especies de murciélagos habitan las cuevas del Parque Nacional Tingo María (PNTM) y cómo se distribuyen dentro de las mismas categorizándolas en tipos de percha. Evalué seis formaciones rocosas en el PNTM en los años 2015 y 2017. Usé redes de niebla y de mano para capturar a los murciélagos. En total registré 16 especies de murciélagos pertenecientes a las familias Phyllostomidae, Emballonuridae y Mormoopidae. En la cueva de "Las lechuzas" encontré el mayor número de especies (10). Categoricé tres tipos de percha usados por los murciélagos dentro de las cuevas: (I) pequeñas bóvedas formadas como depresiones en el techo, (II) espacios angostos en medio de las estalactitas, (III) cavidades amplias entre las estalactitas y la pared en forma de campana. Las cuevas del PNTM contribuyen a la persistencia local de los murciélagos proveyéndoles de refugios, y existe la necesidad de conocer sobre los aspectos ecológicos y la importancia funcional de los murciélagos dentro de estas cuevas.

Palabras clave: Cuevas, Emballonuridae, Mormoopidae, Phyllostomidae, refugios diurnos.

\begin{abstract}
In Peru, there is little information on the biodiversity of subterranean ecosystems, particularly caves. In this work, I recorded which bat species inhabit the caves of the Parque Nacional Tingo María (PNTM) and how they are distributed within them, categorizing them into types of roost. I evaluated six rock formations at PNTM in 2015 and 2017. To capture bats I used mist nets and hand nets. I registered 16 species of bats belonging to the families Phyllostomidae, Emballonuridae, and Mormoopidae. In the cave of "Las lechuzas" I found the greatest number of species (10). I categorized three types of roost used by bats inside caves: (I) small vaults formed as depressions in the ceiling, (II) narrow spaces in the middle of the stalactites, (III) wide cavities between the stalactites and the shaped bell wall. The PNTM caves contribute to the local persistence of bats by providing them with shelter, and there is a need to learn about the ecological aspects and functional importance of bats within these caves.
\end{abstract}

Key words: Caves, diurnal roost, Emballonuridae, Mormoopidae, Phyllostomidae. 
Las cuevas o cavernas, definidas como cavidades subterráneas en una variedad de estratos, son ambientes con altos niveles de biodiversidad (Gunn 2004; Medellin et al. 2017). Esta diversidad influye en el funcionamiento de los ecosistemas circundantes (Medellin et al. 2017). Por ejemplo, especies de murciélagos que utilizan las cuevas proporcionan importantes funciones ecológicas como la polinización, la dispersión de semillas y la reducción de plagas de insectos (Aziz et al. 2017; Bumrungsri et al. 2013; Medellin et al. 2017; Wanger et al. 2014; Sritongchuay et al. 2016). Los ecosistemas subterráneos suelen ser subrepresentados en la mayoría de los estudios ecológicos, planes de conservación y áreas protegidas (Sugai et al. 2015), a pesar que en muchos casos las actividades humanas amenazan estos ecosistemas y a las especies dentro de ellos (Baker \& Genty 1998; Mickleburgh et al. 2002; Medellin et al. 2017). En el Perú la información sobre este tema es escasa (Bowles et al. 1979; Mejia 2013). En 1965 el gobierno peruano creó el Parque Nacional Tingo María (PNTM) con el objetivo principal de proteger la cueva de "Las Lechuzas" (Dourojeanni \& Tovar 1974), uno de los pocos reconocimientos a la importancia de estos ecosistemas en el Perú. En este trabajo se busca determinar qué especies de murciélagos habitan las formaciones rocosas (cuevas, cavernas) dentro del PNTM y como se distribuyen dentro de las mismas, categorizándolas en tipos de percha.

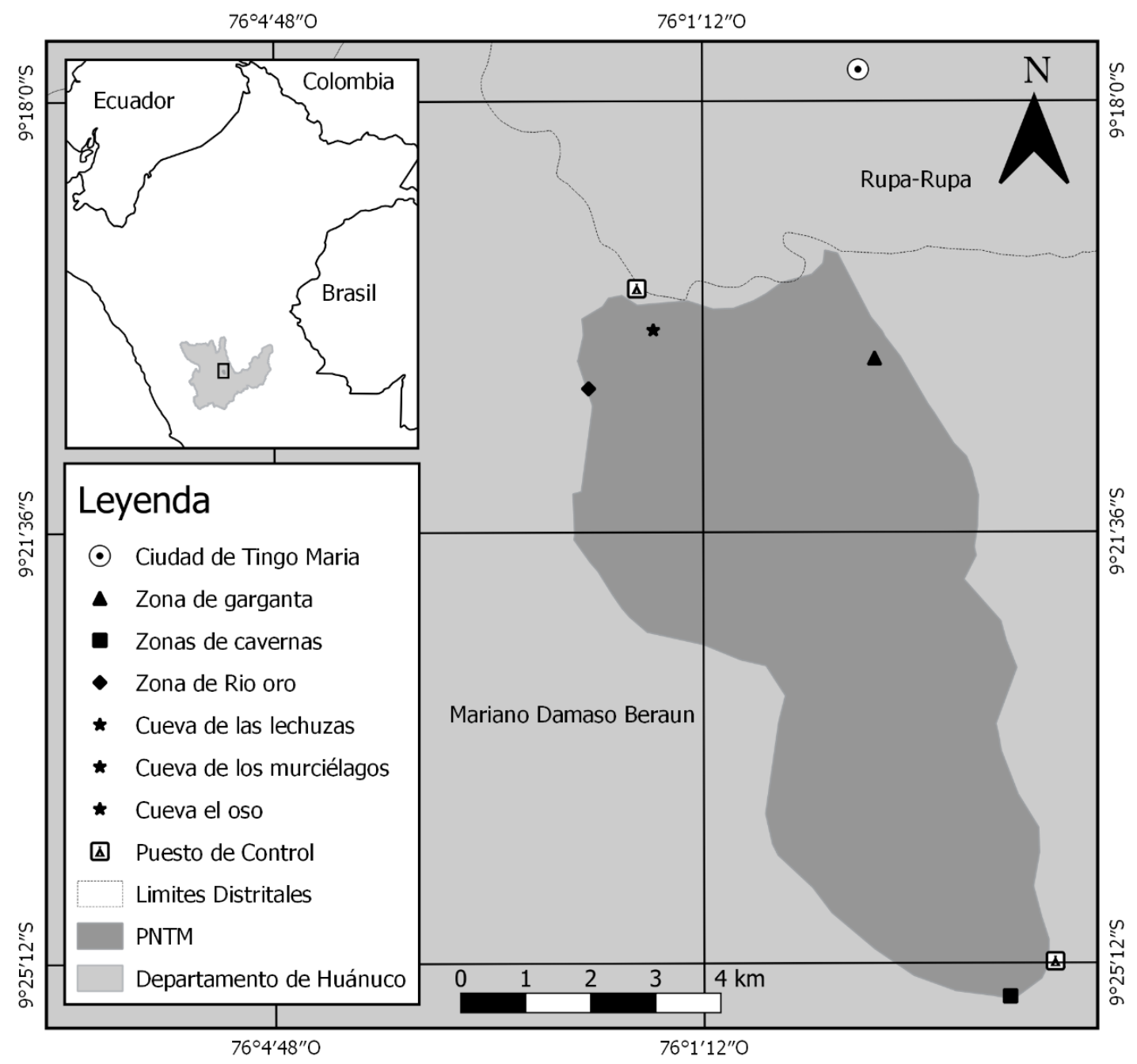

FIGURA 1. Ubicación de las zonas de estudio en el Parque Nacional Tingo María, Huánuco, Perú. 
El presente estudio lo realicé dentro del PNTM, ubicado en el departamento de Huánuco, Perú (Figura 1). El PNTM está formado por una cadena montañosa de piedra caliza cubierta por bosques montanos lluviosos y nublados, entre los 650 y 1.808 msnm. El PNTM abarca 4.777 ha, de las cuales 4.472 ha representan bosque sin alteración por actividad humana (SERNANP 2012). Dentro de caminos ya existentes se buscaron formaciones rocosas (cavernas, cuevas) que estaban siendo usadas como refugios diurnos por los murciélagos. Se identificaron tres zonas de "Garganta", "Río Oro", "Cavernas", y tres cuevas: "Las Lechuzas", "Los Murciélagos", y "El Oso" (Figura 1). Las zonas de "Garganta", "Río Oro" y "Cavernas" son pequeños afloramientos kársticos ubicados en los bordes de los caminos. Mientras, las cuevas de "Las Lechuzas", "El Oso" y "Los Murciélagos" son formaciones kársticas más complejas ubicadas una por encima de la otra en la misma montaña. No se pudo encontrar conexión entre las últimas por lo que se consideraron independientes una de la otra. Las cuevas de "Las Lechuzas" y "El Oso" tienen la particularidad de albergar aves "guacharos"de la especie Steatornis caripensis.

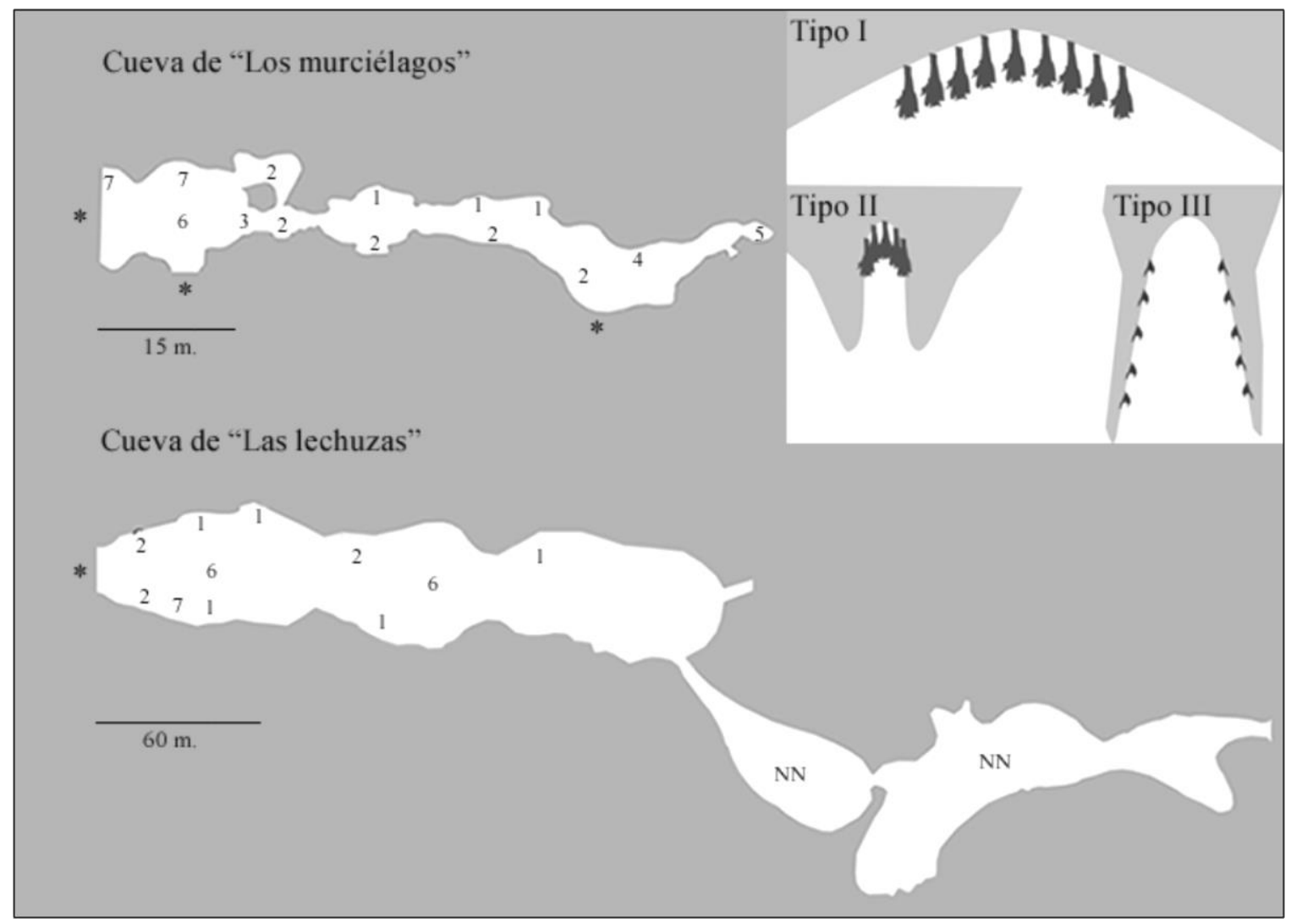

FIGURA 2. Disposición espacial de los murciélagos en las cuevas y tipos de percha. Artibeus sp. (1), Carollia (2), Hsunycteris thomasi (3), Diaemus youngii (4), Desmodus rotundus (5), Phyllostomus hastatus (6), Peropteryx macrotis (7), Especie no identificada (NN).

Las capturas de murciélagos las realicé entre enero y marzo del 2015 y julio del 2017. En el 2015 visité todas las zonas de estudio, una zona por día, durante la mañana desde las 10:00 hasta las 12:00 horas. El 2015 y 2017 evalué la cueva de "Las Lechuzas" y zona de "Cavernas" por tres noches cada una de 17:30 a 20:30 horas, debido a que forman parte de un circuito turístico. Capturé los murciélagos con la ayuda de redes de niebla colocadas en las entradas de las cuevas, también usé redes de mano para capturar los murciélagos al ubicar sus lugares de percha. Para determinar que especies estaban presentes, todos los 
individuos capturados fueron colocados en bolsas de tela para posteriormente determinar su especie con la ayuda de las claves taxonómicas de Gardner 2008, Díaz et al. (2016) y Lopez-Baucells et al. (2016). Debido a que los murciélagos se encontraban en una época reproductiva, al momento de su captura la manipulación de todos los individuos la realicé en el menor tiempo posible siguiendo los protocolos propuestos por Sikes \& ACUC (2016). Adicionalmente busqué de manera oportuna los lugares que los murciélagos utilizaban para percharse durante el día dentro de las formaciones rocosas y las categoricé en tipos (Tipo I: pequeñas bóvedas formadas como depresiones en el techo, Tipo II: espacios angostos en medio de las estalactitas, Tipo III: cavidades amplias entre las estalactitas y la pared en forma de campana) (Figura 2).

Se identificaron 16 especies de murciélagos usando las formaciones rocosas del PNTM, pertenecientes a las familias Emballonuridae (3 especies), Phyllostomidae (12), y Mormoopidae (1). La cueva de "Las Lechuzas", la más grande de las cuevas evaluadas, presentó el mayor número de especies (10) (Tabla 1), el mayor número de murciélagos observados estuvo concentrado dentro de la primera cámara (Figura 2).

TABLA 1. Número de individuos capturados y tipo de percha de los murciélagos cavernícolas en el Parque Nacional Tingo María. *Especies de las que no se registró el tipo de percha usado, y en () el número de individuos capturados en el 2015 y 2017. I, II, III Tipos de percha.

\begin{tabular}{|c|c|c|c|c|c|c|}
\hline ESPECIES & $\begin{array}{c}\text { Cueva de las } \\
\text { Lechuzas }\end{array}$ & $\begin{array}{l}\text { Cueva } \\
\text { el Oso }\end{array}$ & $\begin{array}{l}\text { Cueva de los } \\
\text { Murciélagos }\end{array}$ & $\begin{array}{l}\text { Zona de } \\
\text { Cavernas }\end{array}$ & $\begin{array}{l}\text { Zona de } \\
\text { Garganta }\end{array}$ & $\begin{array}{c}\text { Zona } \\
\text { Rio Oro }\end{array}$ \\
\hline \multicolumn{7}{|l|}{ FAMILIA EMBALLONURIDAE } \\
\hline Saccopterix leptura & & & & III (1) & & \\
\hline Peropteryx kappleri & & & & III (7) & & III (3) \\
\hline Peropteryx macrotis & III (1) & & III (6) & *(5) & & \\
\hline \multicolumn{7}{|l|}{ FAMILIA PHYLLOSTOMIDAE } \\
\hline Carollia perspicillata & III (8) & III (1) & III (4) & I (1) & & \\
\hline Carollia brevicauda & III (3) & & III (1) & I (3) & & III (1) \\
\hline Desmodus rotundus & & & I (1) & & & \\
\hline Diaemus youngii & & & I (1) & & & \\
\hline Hsunycteris thomasi & $*(1)$ & & I (1) & & I (1) & \\
\hline Micronycteris sp & & & & I (1) & & \\
\hline Micronycteris minuta & $*(1)$ & & & *(1) & & \\
\hline Lonchorhina aurita & *(3) & & & & & \\
\hline Phyllostomus hastatus & II (3) & & $\|(1)$ & II (4) & & \\
\hline Artibeus planirostris & II (6) & & $\|(1)$ & II (9) & & \\
\hline Artibeus lituratus & II (1) & & & & & \\
\hline Platyrrhinus infuscus & & & & & I (8) & \\
\hline \multicolumn{7}{|l|}{ FAMILIA MORMOOPIDAE } \\
\hline Pteronotus rubiginosus & * $(8)$ & & & & & \\
\hline TOTAL DE ESPECIES: 16 & 10 & 1 & 8 & 9 & 2 & 1 \\
\hline
\end{tabular}


En las cuevas: de "Las Lechuzas" y "Los Murciélagos" (Figura 2) las especies de los géneros Artibeus, Carollia, Phyllostomus, y Peropteryx se encontraron perchadas principalmente en las cámaras más próximas a las entradas de las cuevas, formando grupos pequeños y dispersos de entre 5 a 10 individuos aproximadamente a excepción de Phyllostomus que formó grupos más numerosos y cercanos que sumaban 100 individuos aproximadamente. Las estalactitas presentes a una altura de 2 a 5 metros eran usadas por los individuos de Artibeus sp., mientras que los individuos de Phyllostomus hastatus usaban las estalactitas que se encontraban en las partes más altas. Las especies de Carollia se percharon mayormente en las paredes laterales a una altura de 3 metros aproximadamente. Se encontró una colonia de Desmodus rotundus en la parte final de la cueva "Los Murciélagos" (Figura 2). No ubiqué los espacios usados para perchar por Micronycteris minuta, Lonchorhina aurita, y Pteronotus rubiginosus dentro de las cuevas. Las especies de mayor tamaño (A. planirostris, A. lituratus y P. hastatus) utilizaron una percha de tipo II, mientras que las especies con tamaño menor utilizaron perchas tipo I (Carollia perspicillata, C. brevicauda, Desmodus rotundus, Diaemus youngii, Hsunycteris thomasi, Micronycteris sp., Platyrrhinus infuscus). Las especies de la familia Emballonuridae solo utilizaron perchas de tipo III. La distinta disposición de los murciélagos dentro de las cuevas podría sugerir que existen condiciones microclimáticas para cada especie, así como una variación en tolerancia a la luz y a la baja concentración de oxígeno según la especie.

En la cueva de "Las Lechuzas" Bowles et al. (1979) también registraron 10 especies, pero seis especies fueron diferentes a las reportadas en este trabajo (Pteronotus personatus, Chrotopterus auritus, Glossophaga soricina, Lonchophylla robusta, Desmodus rotundus, y Diaemus youngi). Debido a que este estudio fue limitado a ciertos meses del año, es necesario realizar evaluaciones mensuales a lo largo del año para poder conocer de manera más completa la riqueza de especies y su dinámica en el uso de las cuevas.

A diferencia de lo observado para los murciélagos del género Artibeus, Kunz et al. (1983) describen que en Puerto Rico A. jamaicensis usa las cavidades, como las estalactitas, distribuyéndose en los bordes y no la parte más profunda (Figura 2, Tipo II). Por otro lado, al igual que mis resultados Trajano \& Giménez (1998) registran que Carollia perspicillata en Brasil usualmente se distribuye cerca a la entrada de la cueva. En el caso de Phyllostomus hastatus se observó que formaban grupos numerosos de al menos 100 individuos en la parte alta del techo dentro de las cuevas en el PNTM, lo cual coincide con los registros de Goodwin \& Greenhall (1961), en Trinidad y Tobago, quienes encontraron que los individuos de esta especie se concentran formando grupos grandes. También ha sido reportado que algunas especies de la familia Emballonuridae suelen refugiarse en lugares bien iluminados, perchándose a baja altura y cerca a la entrada (Goodwin \& Greenhall 1961). Este comportamiento se observó en Peropteryx macrotis, Peropteryx kappleri y Saccopterix leptura dentro del PNTM. En el caso de Lonchorhina aurita, según Emmons \& Feer (1997) cuenta con una distribución limitada a áreas con cuevas o rocas, mientras que Nelson (1965) y Bloedel (1955) sugieren que se refugia en grietas inaccesibles formando grupos densos en las partes altas del techo (Goodwin \& Greenhall 1961). A pesar de esto, no se pudo encontrar el lugar utilizado por L. aurita para percharse dentro de la cueva de "Las Lechuzas".

Considerando que en Perú son pocas las cuevas que presentan una gran riqueza de murciélagos, el PNTM representa uno de los sitios más importantes en la conservación de estas especies en Perú. Las cuevas de esta área natural contribuyen a la persistencia local de los murciélagos, ya que son utilizadas por diferentes especies como sitios de descanso, 
reproducción y refugios permanentes. Existe la necesidad de generar más conocimiento sobre los aspectos ecológicos y la importancia funcional de los murciélagos presentes en cuevas.

\section{AGRADECIMIENTOS}

A los guardaparques, especialistas y voluntarios del Parque Nacional Tingo y a los alumnos de la facultad de Recursos Renovables de la Universidad Nacional Agraria de la Selva por su ayuda en campo, y a Farah Carrasco por su consejo y guía.

\section{REFERENCIAS}

Aziz SA, Clements GR, McConkey KR, Sritongchuay T, Pathil S, Abu Yazid MNH, Campos-Arceiz A, Forget PM, Bumrungsri S. 2017. Pollination by the locally endangered island flying fox (Pteropus hypomelanus) enhances fruit production of the economically important durian (Durio zibethinus). Ecology and evolution 7:8670-8684. https:// doi.org/10.1002/ ece3.3213

Baker A, Genty D. 1998. Environmental pressures on conserving cave speleothems: effects of changing surface land use and increased cave tourism. Journal of Environmental Management 53:165-175. https:// doi.org/10.1006/jema.1998.0208

Bloedel P. 1955. Observations on the life histories of Panama bats. Journal of Mammalogy 36:232235. https://doi.org/10.2307/1375881

Bowles JB, Cope JB, Cope EA. 1979. Biological studies of selected Peruvian bats of Tingo Maria, Departamento de Huánuco. Transactions of the Kansas Academy of Science 1903:1-10. https:// doi.org/10.2307/3627013

Bumrungsri S, Lang D, Harrower C, Sripaoraya E, Kitpipit K, Racey PA. 2013. The dawn bat, Eonycteris spelaea Dobson (Chiroptera: Pteropodidae) feeds mainly on pollen of economically important food plants in Thailand. Acta Chiropterologica 15:95-104. https:// doi.org/10.3161/150811013X667894

Díaz MM, Solari S, Aguirre LF, Aguiar LM, Barquez RM. 2016. Clave de identificación de los murciélagos de Sudamérica-Chave de identificacao dos morcegos da America do Sul. Publicación Especial Número 2.

Dourojeanni MD, Tovar A. 1974. Notas sobre el ecosistema y la conservación de la Cueva de las Lechuzas (Parque Nacional de Tingo María, Perú). Revista Forestal del Perú 5:1-2. http:// dx.doi.org/10.21704/rfp.v5i1-2.1092

Emmons L, Feer F. 1997. Neotropical rainforest mammals: A field guide. 2nd ed. Chicago: The University of Chicago Press.

Gardner AL. (Ed.). 2008. Mammals of South America, volume 1: marsupials, xenarthrans, shrews, and bats (Vol. 1). University of Chicago Press, Chicago.

Goodwin GG, Greenhall AM. 1961. A review of the bats of Trinidad and Tobago: descriptions, rabies infection, and ecology. Bulletin of the AMNH; v. 122, article 3.

Gunn J. (Ed.). 2004. Encyclopedia of caves and karst science. Taylor \& Francis, New York.

Kunz TH, August PV, Burnett CD. 1983. Harem social organization in cave roosting Artibeus jamaicensis (Chiroptera: Phyllostomidae). Biotropica 15:133-138. http://dx.doi.org/10.2307/2387958 
Lopez-Baucells A, Rocha R, Bobrowiec PED, Palmeirim JM, Meyer CFJ. 2016. Field guide to Amazonian Bats. Editorial INPA, Manaus.

Medellin RA, Wiederholt R, Lopez-Hoffman L. 2017. Conservation relevance of bat caves for biodiversity and ecosystem services. Biological conservation 211:45-50. https://doi.org/10.1016/j.biocon.2017.01.012

Mejia FM. 2013. Biodiversidad de artrópodos y su relación con Steatornis caripensis Humboldt "Guácharo" con fines de conservación. Tingo María-Perú. Tesis para optar el grado de Maestro de Ciencias. Universidad Nacional Agraria de la Selva.

Mickleburgh SP, Hutson AM, Racey PA. 2002. A review of the global conservation status of bats. Oryx 36:18-34. https:// doi.org/10.1017/S0030605302000054

Nelson CE. 1965. Lonchorhina aurita and other bats from Costa Rica. Texas Journal of Science 17:303.

SERNANP (Servicio Nacional de Áreas Naturales Protegidas por el Estado). 2002. Plan Maestro 20122017 del Parque Nacional Tingo María, Tingo María-Perú.

Sikes RS. ACUC (Animal Care and Use Committee of the American Society of Mammalogist, United States of America). 2016. Guidelines of the American Society of Mammalogists for the use of wild mammals in research and education. Journal of Mammalogy 97:663-688. https://doi.org/10.1093/jmammal/gyw078

Sritongchuay T, Kremen C, Bumrungsri S. 2016. Effects of forest and cave proximity on fruit set of tree crops in tropical orchards in Southern Thailand. Journal of Tropical Ecology 32:269-279. https:// doi.org/10.1017/S0266467416000353

Sugai LSM, Ochoa-Quintero JM, Costa-Pereira R, Roque FO. 2015. Beyond above ground. Biodiversity and Conservation 24:2109-2112. https:// doi.org/10.1007/s10531-015-0918-4

Trajano E, Gimenez EE. 1998. Bat community in a cave from eastern Brazil, including a new record of Lionycteris (Phyllostomidae, Glossophaginae). Studies on Neotropical Fauna and Environment 33:69-75. https:// doi.org/10.1076/snfe.33.2.69.2156

Wanger TC, Darras K, Bumrungsri S, Tscharntke T, Klein AM. 2014. Bat pest control contributes to food security in Thailand. Biological Conservation 171:220-223. https://doi.org/10.1016/j.biocon.2014.01.030

Editor: Diego J. Lizcano Recibido: 2020-06-11 Revisado: 2020-07-14 Aceptado: 2020-07-17 Publicado: 2020-09-28 